\title{
Temporal Dysynchrony in brain connectivity gene expression following hypoxia
}

\author{
Brett Milash', Jingxia Gao², Tamara J. Stevenson², Jong-Hyun Son², Tiffanie Dahl² and Joshua L. Bonkowsky ${ }^{2,3^{*}}$
}

\begin{abstract}
Background: Despite the fundamental biological importance and clinical relevance of characterizing the effects of chronic hypoxia exposure on central nervous system (CNS) development, the changes in gene expression from hypoxia are unknown. It is not known if there are unifying principles, properties, or logic in the response of the developing CNS to hypoxic exposure. Here, we use the small vertebrate zebrafish (Danio rerio) to study the effects of hypoxia on connectivity gene expression across development. We perform transcriptional profiling at high temporal resolution to systematically determine and then experimentally validate the response of CNS connectivity genes to hypoxia exposure.

Results: We characterized mRNA changes during development, comparing the effects of chronic hypoxia exposure at different time-points. We focused on changes in expression levels of a subset of 1270 genes selected for their roles in development of CNS connectivity, including axon pathfinding and synapse formation. We found that the majority of CNS connectivity genes were unaffected by hypoxia. However, for a small subset of genes hypoxia significantly affected their gene expression profiles. In particular, hypoxia appeared to affect both the timing and levels of expression, including altering expression of interacting gene pairs in a fashion that would potentially disrupt normal function.

Conclusions: Overall, our study identifies the response of CNS connectivity genes to hypoxia exposure during development. While for most genes hypoxia did not significantly affect expression, for a subset of genes hypoxia changed both levels and timing of expression. Importantly, we identified that some genes with interacting proteins, for example receptor/ligand pairs, had dissimilar responses to hypoxia that would be expected to interfere with their function. The observed dysynchrony of gene expression could impair the development of normal CNS connectivity maps.
\end{abstract}

Keywords: Hypoxia, Connectivity, Synapse, Axon pathfinding, Zebrafish

\section{Background}

Hypoxic injury complicates up to $60 \%$ of preterm births and leads to a broad range of neurological birth defects including epilepsy, autism, ADHD, and mental retardation [1]. Despite the significant clinical impact, the specific effects of hypoxia on the developing central nervous system (CNS) are poorly characterized, and the molecular mechanisms largely unknown. Imaging studies show altered patterns of connectivity in brain magnetic resonance imaging (MRI) of children born prematurely $[2,3]$. The timing of

\footnotetext{
* Correspondence: joshua.bonkowsky@hsc.utah.edu

${ }^{2}$ Department of Pediatrics, University of Utah, 295 Chipeta Way, 84108 Salt Lake City, UT, USA

${ }^{3}$ Department of Neurobiology and Anatomy, University of Utah School of Medicine, Salt Lake City, UT, USA

Full list of author information is available at the end of the article
}

preterm birth, from 24 up to 37 weeks gestation, encompasses a key CNS developmental window characterized by widespread axon pathfinding and synaptogenesis, and thus makes the brain particularly vulnerable to disruptions of connectivity. Major pathways established during this at-risk period in premature infants include the extension of the corticospinal tracts, the development of the corpus callosum, and the formation of connections in the cortical hemispheres [4-6]. Development of connectivity in the CNS is a critical step in neural development and appears to be disrupted in disorders including autism and intellectual disability [7-9]. The development of connectivity more precisely involves the processes of axon guidance and pathfinding, synapse development and stabilization; and in the mature CNS, circuit properties and functional networks $[10,11]$. 
Experimental studies have demonstrated that developmental hypoxia can disrupt axon pathfinding $[12,13]$ and alter synapse gene expression [14]. hifl $\alpha$, a basic helixloop-helix transcription factor whose protein expression is stabilized in hypoxia, controls many of the downstream responses to hypoxia including in the CNS [15]. In addition, hif1 $\alpha$ is necessary for normal CNS development [16], although its precise role is unclear. A small number of target genes involved in CNS connectivity and dysregulated by hypoxia have been identified and experimentally validated; including adenosine A2 receptor, Brn3b, EphrinB2, Netrin-4, and VAB-1 [12, 13, 17-19].

However, a global understanding of the changes in the genetic regulatory landscape of the developing CNS connectome caused by hypoxia is lacking. Developmentally, it is not known if there are critical hypoxia exposure times. Functionally, it is unclear if certain types of connectivity genes are more affected by hypoxia, and what classes or types of genes are at risk. To address these questions in a comprehensive, reproducible, and experimentally accessible fashion we performed our experiments in the small vertebrate zebrafish (Danio rerio). Advantages of zebrafish are that it combines the relevancy of vertebrate CNS structures and genes, with rapidity and efficiency for testing molecular mechanisms. Its low cost and ability to generate large numbers of animals in a range of experimental conditions provide significant statistical power. In addition, fundamental developmental, neurobiological, and genomic mechanisms are conserved with mammals [20-22].

Our objective was to identify the gene expression changes occurring with hypoxia in the developing CNS, and to determine if there were patterns of gene expression changes that would inform understanding of the effects of hypoxia. Our focus was on characterizing the effects of hypoxia on connectivity genes. We compared gene expression profiles across development during the first three days of zebrafish embryogenesis, when the majority of CNS development occurs, including neurogenesis, axon pathfinding, and synapse formation. Analysis was limited to those genes involved in CNS connectivity development. We found that the majority of CNS connectivity genes were unaffected by hypoxia. However, for a small subset of genes hypoxia significantly affected their expression profiles, and caused changes in both timing and levels of expression that could have significant effects on connectivity development.

\section{Results and Discussion}

\section{Studying the effects of hypoxia on CNS connectivity development}

To study the effects of hypoxia on development of the $\mathrm{CNS}$, and in particular the effects of chronic hypoxia on the formation of connectivity in the CNS, we assayed for changes in gene expression profiles. We collected total RNA from zebrafish at five different embryonic developmental stages ( $24 \mathrm{~h}$ post-fertilization (hpf), $36 \mathrm{hpf}, 48 \mathrm{hpf}$, $60 \mathrm{hpf}, 72 \mathrm{hpf}$ ): the five stages reflect key epochs in early zebrafish development, and encompass the majority of CNS connectivity development, from neurogenesis through synapse formation (Fig. 1). For the majority of our subsequent analyses, we restricted our analysis to 1270 genes with known or likely roles in the development of CNS connectivity (Fig. 1; Additional file 1).

\section{Identification and cluster analysis of differentially expressed genes}

After read mapping, gene expression was calculated using DESeq2 [23] and the abundances of genes were expressed as FPKM (fragments per kilobase of transcript per million fragments mapped). Principal component analysis (PCA) using the entire transcriptome of 31,860 genes and performed with both the normoxia and hypoxia data sets showed some separation of samples in

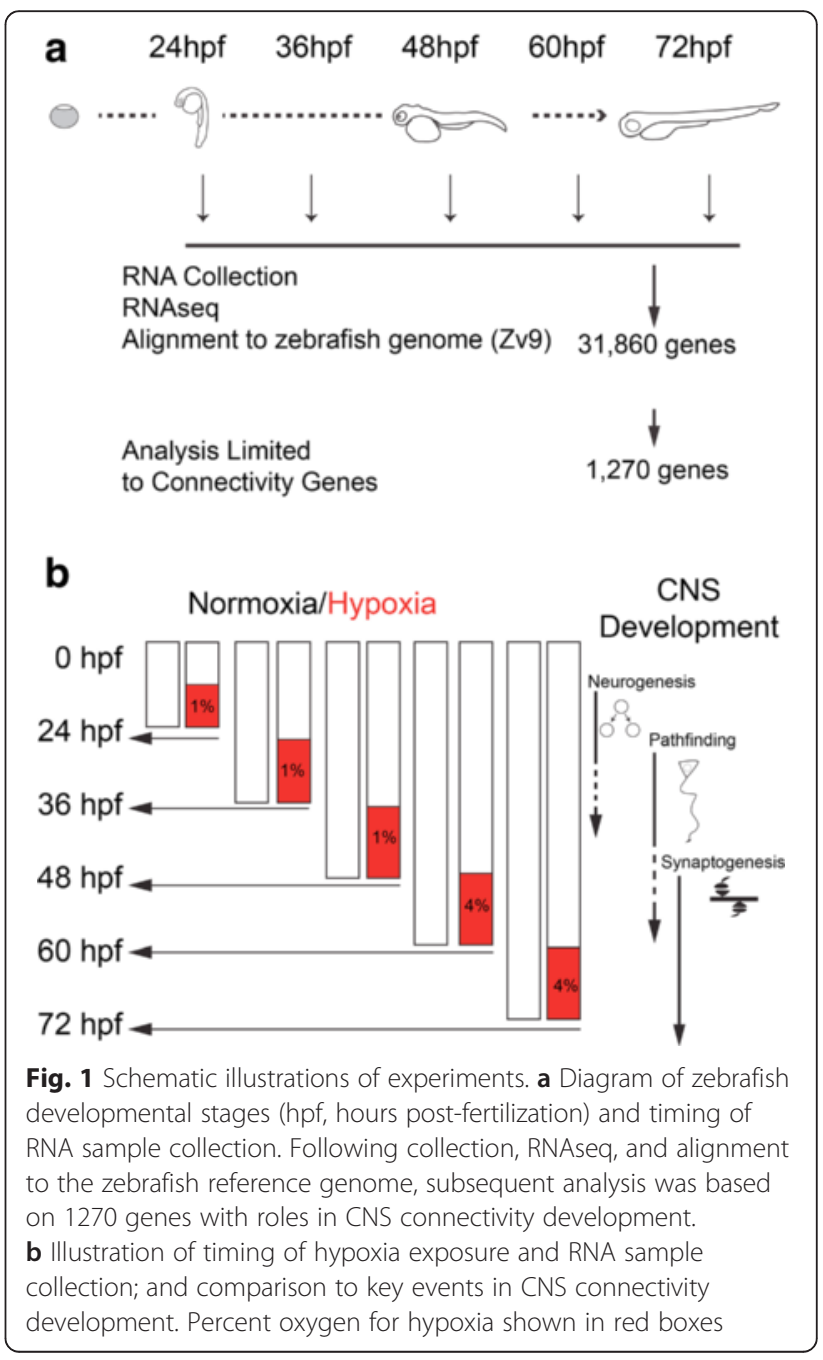


earlier developmental stages, but tighter clustering at older ages (Fig. 2a). This trend remained when the normoxic and hypoxic samples were analyzed separately (Fig. 2b, c). The PCA results show that the RNAseq read variance for the experimental replicates at any given developmental stage or experimental condition (hypoxia or normoxia) were more similar to each other than to samples from different ages or conditions. Thus, the PCA shows that the different conditions (developmental stage; hypoxia/normoxia) were the contributors for the variance.

\section{Expression analysis across development}

Gene expression analysis across development during normoxia showed that the 1270 genes fell into ten Kmeans clusters (Fig. 3a-d). Differentially expressed genes, compared to their average expression across development, were defined as genes with a false discovery rate (FDR) less than 0.1 and a more than a 2-fold expression change (Additional file 2).

\section{Expression analysis comparing hypoxia and normoxia and across development}

For hypoxia, the relative expression of the genes was compared to their normoxic levels (Fig. 3e-h; Additional file 3; Additional file 4). To adjust for changes in levels due to changes associated with development, we normalized expression for each gene to its mean level across development in each experimental condition (normoxic or hypoxic), and then compared hypoxic to normoxic levels at each developmental time-point (Fig. 4a; Additional file 5). We found that the 1270 genes grouped into fourteen K-means clusters. The largest cluster (223 genes), cluster 1, had minimal changes across development or with hypoxic conditions. Several other clusters showed a developmental increase in gene expression but with little or no effect from hypoxia (clusters $2,7,10,12$; total of 526 genes). In total 749 genes (59 \%) were unaffected by hypoxia. The remaining clusters demonstrated differential responses of gene expression to hypoxia during development. Expression analysis showing three representative genes from clusters 5, 8, and 14 displayed as lines demonstrates how different genes had differential responses to hypoxia across development (Fig. 4b). Interestingly, Clusters 5 and 11, which displayed the most dynamic responses to hypoxia, contained a total of only nine genes. However, although transcription factors constituted a majority of the two clusters (Fig. 4c, d; Additional file 6), there were not other apparent shared biological features of the clusters, including a Pathway Commons Network analysis showing no direct protein-protein interactions between the members of clusters 5 or 11 (Fig. 4e) [24]. GO pathway analysis was most descriptive for cluster 1 , the largest cluster; while clusters 2,7 , and 10 , which showed increases in expression during development but no effect from hypoxia, contained a higher proportion of receptor/ligand genes (Fig. 4f). Thus, there did not appear to be a certain type or category of genetic response to hypoxia, as least as tested with Pathway Commons Network analysis or GO pathway analysis.

We also tested whether analysis of the entire original transcriptome $(n=31,860)$ would result in an alternative pattern of clustering of expression changes. K-means analysis of the entire transcriptome resulted in eighteen clusters. Genes that clustered together in the connectivity gene analysis were often split into different clusters when the entire transcriptome was analyzed (Fig. 5; Additional file 7). The $\mathrm{K}$ cluster analysis for the entire transcriptome often provided decreased resolution compared to when only connectivity genes were analyzed. For example, some genes from clusters 5 and 9 in the pan-transcriptome analysis, when analyzed in the connectivity gene set could be observed to in fact have differential expression profiles at 24 and $48 \mathrm{hpf}$ (Fig. 5b, red lines). However, it is reassuring that for most genes their cluster assignments and grouping remained
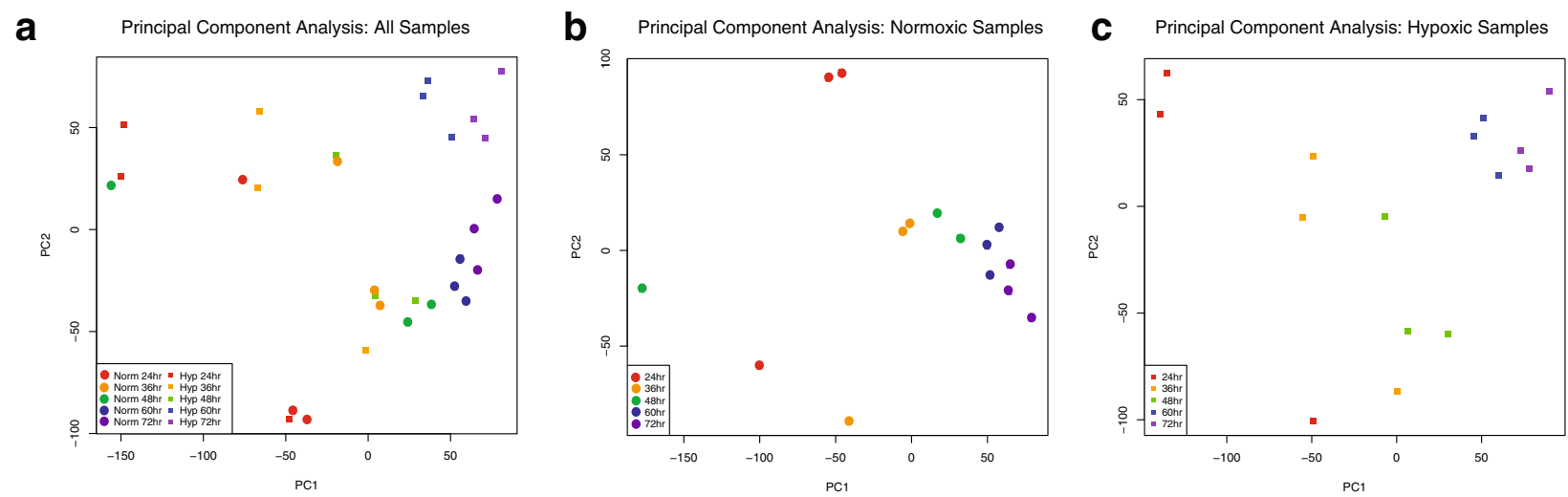

Fig. 2 Principal Component Analysis of Gene Expression. Principal component analysis of entire data set ( $n=31,860$ transcripts) shows closer clustering at later time stages (60 and $72 \mathrm{hpf}$ ), but more spread at earlier time points. Separate analysis of normoxic-only or hypoxic-only data sets displays similar findings 


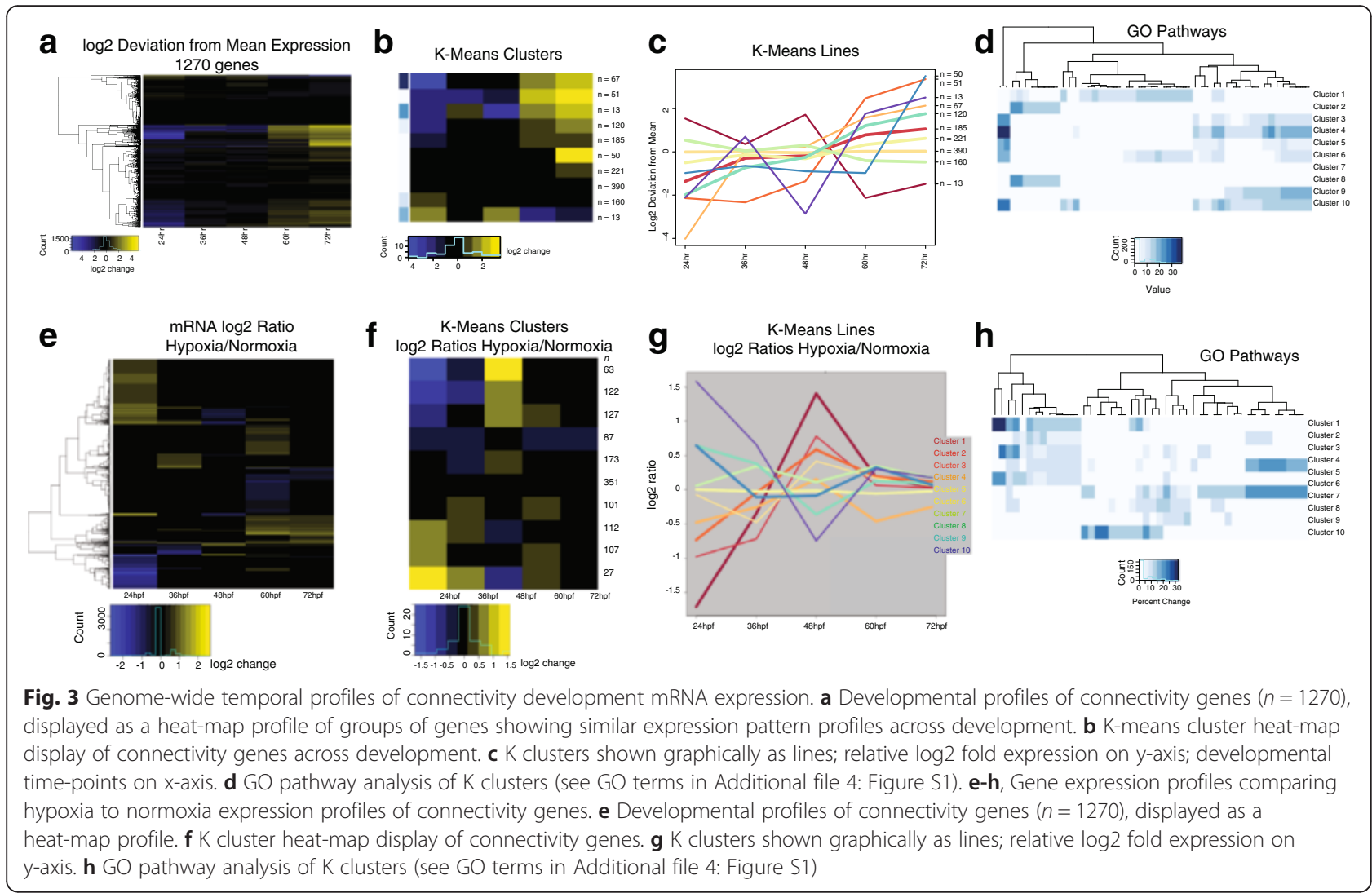

constant in both the whole transcriptome and connectivity-only analyses (Fig. 5c). Of note, analysis of the entire transcriptome included the hypoxia-inducible factor (hif) genes. hif1ab, hif1an, and hif2(epas1a) were in cluster 1; hif2(epas1b) and hif1al2 were in cluster 13; and hiflaa was in cluster 14. Thus, we think that the use of the entire transcriptome for $\mathrm{K}$ means clustering does not provide additional insight and actually obscures some of the findings from analysis of the 1270 connectivity genes.

\section{in vivo testing, $q R T-P C R$ validation, and biological significance}

To perform testing of the RNAseq results and to validate RNAseq results for individual genes, we performed experiments in the embryonic zebrafish and compared hypoxic to normoxic animals. We analyzed expression for three genes from three different clusters by both in situ hybridization and quantitative RT-PCR (Fig. 6a, b; Additional file 8). ryk, a receptor-tyrosine kinase from cluster 1 , has decreased expression at $24 \mathrm{hpf}$ in hypoxia (fold change 0.33 ; two-way $t$ test $p<0.001$ ), but otherwise is relatively constant across development and in normoxia versus hypoxia. nrxn1a, a synaptic transmembrane protein in cluster 9, demonstrates increased expression levels at later developmental stages. However, hypoxia diminishes expression at $24 \mathrm{hpf}$ and $36 \mathrm{hpf}$ (fold changes 0.13 and 0.52 ; two-way t tests $p<0.01$ ). fezf 2 , a transcription factor important in neuronal fate specification in cluster 13, had higher levels of expression in early development. Interestingly hypoxia caused a significant increase in expression levels at $24 \mathrm{hpf}$ (fold change 2.1; two-way $t$ test $p<0.01)$. Thus, for these genes the qRT$\mathrm{PCR}$ results match the in situ results. However, there are a few minor discordances compared to the RNAseq results (Additional file 5); for example for ryk at $24 \mathrm{hpf}$, nrxn1a at $48 \mathrm{hpf}$, and fezf2 at $72 \mathrm{hpf}$, the RNAseq change in hypoxia is discordant with the in situ and qRT-PCR results, emphasizing the importance of followup experimental validation.

The temporal changes demonstrated that hypoxia could affect gene regulation and action by changes in overall expression levels. We then considered whether hypoxia could cause a dysynchrony by altering relative expression of genes known to interact, for example receptor-ligand pairs. A change in relative expression of one or both genes that interact could potentially have additive effects on disrupting signaling. We examined four pairs of genes with potential interactions and roles in connectivity: plxnA3 and sema3ab; $d c c$ and $u n c 5 b$; dlg4 and syn1; and nrxn1a and nlgn1 (Fig. 6c). At $24 \mathrm{hpf}$ $p l x n A 3$ and sema3ab were both up-regulated by hypoxia; 


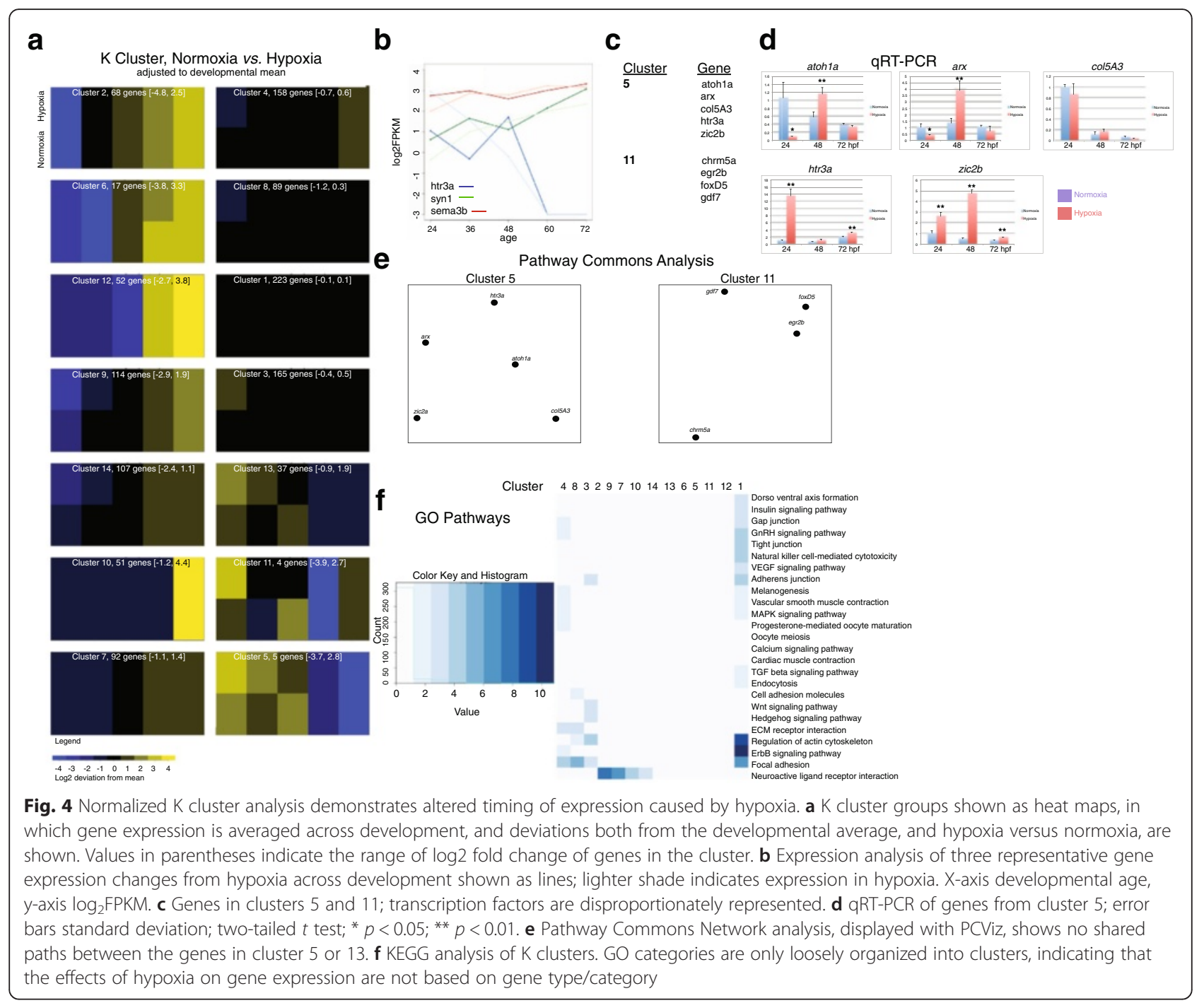

these genes are a cell-surface receptor/ligand pair [25], and increased expression of both genes would disrupt normal axon guidance. $d c c$ and $u n c 5 b$ at $24 \mathrm{hpf}$ are also up-regulated by hypoxia; both genes are receptors for netrin-1 and are necessary for normal midline axon guidance [26]. Examination of the effects of hypoxia at 72 hpf showed minimal effects on nrxn1a and nlgn1, which have roles in synapse adhesion [27]. dlg4, a postsynaptic scaffolding protein, and syn1, a pre-synaptic vesicle-associated protein, show contrasting up- and down-regulation by hypoxia at $72 \mathrm{hpf}$, which could interfere with normal synaptic function.

To test whether the connectivity genes fit into known protein interaction networks we analyzed genes with the most significant changes from hypoxia, while controlling for their expression changes across development (Fig. 7; Additional file 9). The differential expression test was applied to each gene individually, and a $p$ value was determined for each individual gene. Then an adjusted $p$ value was calculated from all the individual $p$ values with adjustment for multiple testing. 57 genes had adjusted $p$ values $<0.05$, but only a minority of this group $(\sim 15)$ had direct protein-protein interactions with each other (Fig. 7a) [28], and KEGG term analysis had sparse representation in a few groups (Fig. 7b), and GO term analysis revealed no statistically significant pathway memberships. 244 genes had unadjusted $p$ values $<0.05$ (no adjustment made for the multiple comparisons), and showed multiple interactions in many KEGG pathways (Fig. 7c, d). An enlarged figure (Additional file 10: Figure S2) shows gene names more clearly for Fig. 7a, c.

When we compared overall gene expression changes with development and with experimental condition, we found that at later ages genes clustered primarily by developmental time, and less by hypoxia versus normoxia (Fig. 7e). In contrast, at earlier ages the relative effects of hypoxia were more significant, and expression changes clustered in a mixture of time and hypoxia effects. With 


\section{$\mathrm{K}$ Clusters, Normoxia vs. Hypoxia
adjusted to developmental mean}

a All Genes $(n=31,860)$

b Connectivity Genes $(n=1,270)$
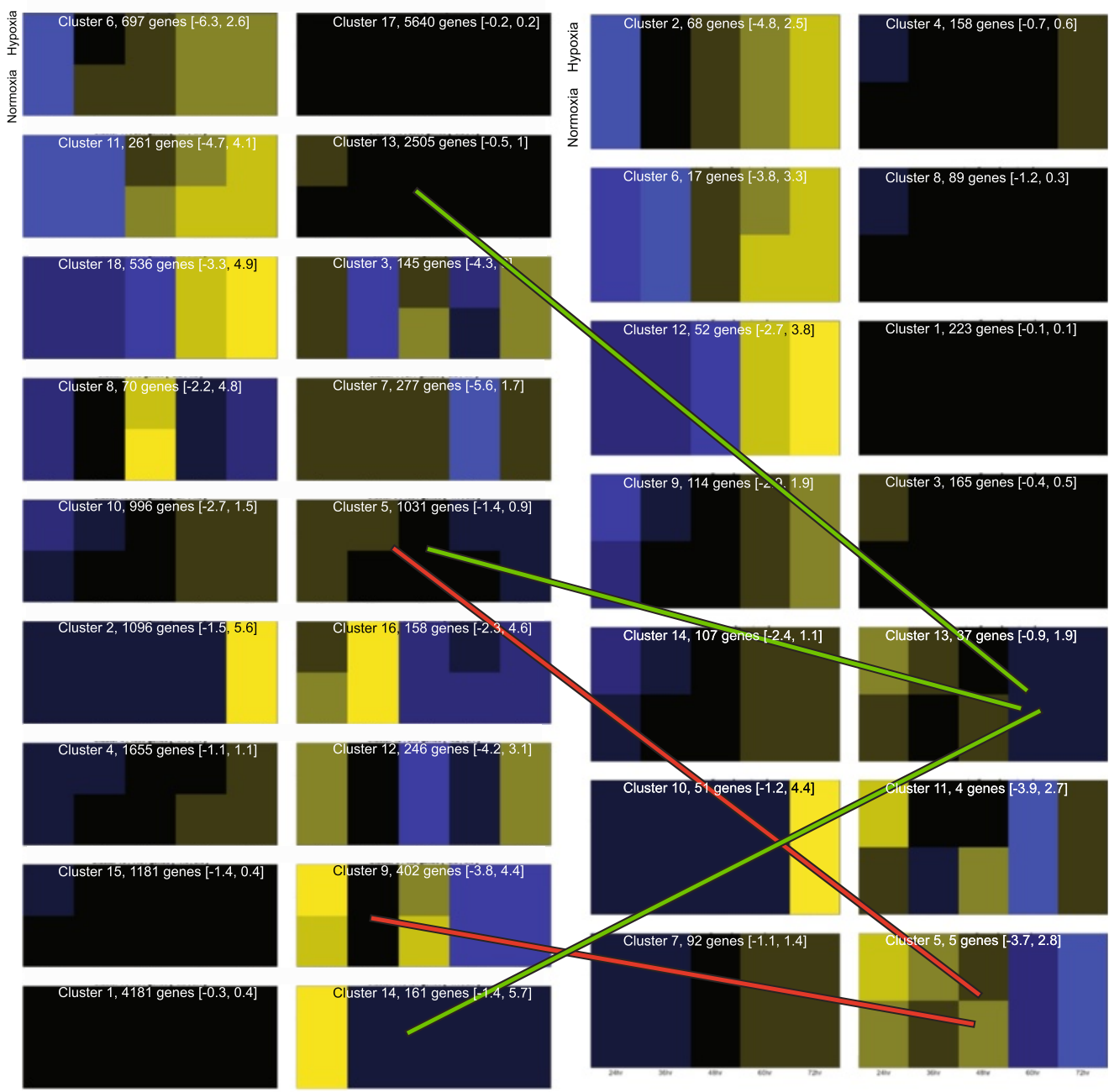

Legend

$\begin{array}{lllllllll}-4 & -3 & -2 & -1 & 0 & 1 & 2 & 3 & 4\end{array}$ Log2 deviation from mean

C

Cluster \#, Entire Transcriptome

\begin{tabular}{|c|c|c|c|c|c|c|c|c|c|c|c|c|c|c|c|c|c|c|c|}
\hline \multirow{10}{*}{ 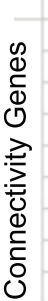 } & & 1 & 2 & 3 & 4 & 5 & 6 & 7 & 8 & 9 & 10 & 11 & 12 & 13 & 14 & 15 & 16 & 17 & 18 \\
\hline & 1 & 84 & 0 & 0 & 0 & 0 & 0 & 0 & 0 & 0 & 0 & 0 & 0 & 7 & 0 & 0 & 0 & 132 & 0 \\
\hline & 2 & 0 & 0 & 0 & 0 & 0 & 68 & 0 & 0 & 0 & 0 & 0 & 0 & 0 & 0 & 0 & 0 & 0 & 0 \\
\hline & 3 & 0 & 0 & 0 & 0 & 18 & 0 & 0 & 0 & 0 & 0 & 0 & 0 & 71 & 0 & 0 & 0 & 76 & 0 \\
\hline & 4 & 92 & 0 & 0 & 48 & 0 & 0 & 0 & 0 & 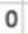 & 0 & 0 & 1 & 0 & 0 & 17 & 0 & 0 & \\
\hline & 5 & 0 & 0 & 0 & 0 & 1 & 0 & 0 & 0 & 4 & 0 & 0 & 0 & 0 & 0 & 0 & 0 & 0 & 0 \\
\hline & 6 & 0 & 0 & 2 & 0 & 0 & 0 & 0 & 0 & 0 & 1 & 14 & 0 & 0 & 0 & 0 & 0 & 0 & 0 \\
\hline & 7 & 1 & 2 & 0 & 80 & 0 & 0 & 0 & 0 & 0 & 9 & 0 & 0 & 0 & 0 & 0 & 0 & 0 & 0 \\
\hline & 8 & 9 & 0 & 0 & 0 & 0 & 0 & 0 & 0 & 0 & 0 & 0 & 0 & 0 & 0 & 74 & 0 & 6 & 0 \\
\hline & 9 & 0 & 0 & 0 & 0 & 0 & 3 & 0 & 0 & 0 & 111 & 0 & 0 & 0 & 0 & 0 & 0 & 0 & 0 \\
\hline$\#$ & 10 & 0 & 50 & 0 & 0 & 0 & 0 & 1 & 0 & 0 & 0 & 0 & 0 & 0 & 0 & 0 & 0 & 0 & 0 \\
\hline 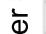 & 11 & 0 & 0 & 1 & 0 & 0 & 0 & 3 & 0 & 0 & 0 & 0 & 0 & 0 & 0 & 0 & 0 & 0 & 0 \\
\hline \pm & 12 & 0 & 0 & 0 & 0 & 0 & 0 & 0 & 0 & 0 & 1 & 0 & 2 & 0 & 0 & 0 & 0 & 0 & 49 \\
\hline 그 & 13 & 0 & 0 & 0 & 0 & 11 & 0 & 0 & 0 & 0 & 0 & 0 & 0 & 25 & 1 & 0 & 0 & 0 & \\
\hline & 14 & 0 & 0 & 0 & 28 & 0 & 0 & 0 & 0 & 0 & 69 & 0 & 0 & 0 & 0 & 10 & 0 & 0 & \\
\hline
\end{tabular}

Fig. 5 (See legend on next page.) 
(See figure on previous page.)

Fig. 5 Normalized K cluster analysis of all genes compared to connectivity genes only shows improved resolution of expression differences. a Analysis of all genes $(n=31,860)$, K cluster groups shown as heat maps, in which gene expression is averaged across development, and deviations both from the developmental average, and hypoxia versus normoxia, are shown. Values in parentheses indicate log 2 range fold change of genes in the cluster. $\mathbf{b}$ Examples of differential assignment of genes to different clusters (heat-maps of connectivity genes is from Fig. 4a). Green lines show different groups of genes assigned to different clusters in the two analyses for cluster 13. Red lines show differential assignment for cluster 5. c Table representation of differences in gene assignment to K clusters, comparing all genes (columns) to connectivity genes only (rows)

this analysis the dysynchrony of expression is more notable- for example, that the gene expression profile of animals at $36 \mathrm{hpf}$ of normoxia is more similar to that of hypoxic animals at $48 \mathrm{hpf}$.

\section{Conclusions}

We combined a high-resolution transcriptional time course analysis, developmental comparisons of expression, and effects of hypoxia, to characterize and validate the role of hypoxia on connectivity gene expression in the CNS. We had several major findings. First, we found that the majority of CNS connectivity genes are not significantly affected by hypoxia during development. Second, for the minority of connectivity genes which are affected by hypoxia do not represent a single type or category of gene or protein. Third, connectivity genes affected by hypoxia display a dysynchrony. That is, their relative expression level is aberrantly increased or decreased at an inappropriate time of development. Hypoxia also altered expression levels at defined developmental times of interacting gene pairs, including axon guidance ligand/receptor pairs and synaptic proteins, that would be predicted to disrupt their normal combinatorial functioning. While following hypoxia embryos showed a slowing in their developmental progression, the gene response to hypoxia was not simply a delay in the timing of their normal expression, and in some cases the hypoxia in fact caused an "acceleration" with increased levels of expression.

Previous work from our group and others has shown that hypoxic injury can disrupt the normal development of axon and synaptic connectivity [12-14, 29]. The work presented here extends those findings by demonstrating that hypoxia acts at least in part by a causing a dysynchronous response of genes including of interacting receptor/ligand pairs. For example, the receptor/ligand pair plxnA3 and sema3ab are both up-regulated by hypoxia, which could lead to elevated GTPase activity and increase repulsive axon guidance [30]. Or, as we found, hypoxia causing a decrease in pre-synaptic syn1 coupled with a post-synaptic increase in $d l g 4$, could alter synaptic stabilization and function [31, 32].

While the changes in gene expression reveal some of the functional logic of the hypoxic response in our analysis we are not able to differentiate direct or indirect effects. Genes could be directly regulated by hypoxia- responsive transcription factors; or could be downstream targets in a multi-step cascade. Our findings provide an excellent starting point for deciphering the regulation of genes by hypoxia. In addition, future experiments can address why certain genes are particularly vulnerable to hypoxia, which has important implications for understanding the effects of hypoxia on CNS connectivity development. In our analysis we focused our attention on a subset of 1270 genes with known roles in connectivity development, in particular on genes with roles in axon pathfinding and synapse development and stabilization. Since the genes regulating connectivity development are relatively well-established [33], our limitation of analysis to this subset provided an enhanced ability to detect trends and patterns that would otherwise have been obscured by including the entire transcriptome.

Future studies could also examine whether certain neuron groups are more susceptible to the effects of hypoxia. While we used RNA from the head and brain, changes in expression from other non-neural tissues could have affected our results. Subsequent studies could use genetically-defined methods to limit analysis to neural tissue only, for example with fluorescenceactivated cell sorting (FACS) analysis or translating ribosomal affinity purification (TRAP) [34].

The transcription factor hif $1 \alpha$ is a master regulator of the cellular response to hypoxia [15] along with a collection of other hif genes and transcription factors [35]. hif1 $\alpha$ is necessary for normal brain development [36], but in the developing brain hypoxia or ectopic expression of hifl $\alpha$ interrupts normal axon guidance [12]. This adverse effect of hypoxia on connectivity has also been observed to affect synapse development in a rodent model [14]. When we analyzed the changes in expression of the different hif isoforms in response to hypoxia we noted relatively minor changes. This is similar to a previous report in zebrafish [37], and is likely due to the regulation of hif by hypoxia predominantly occurring at the post-transcriptional (protein) stage [38].

Chronic hypoxia and injury to the developing brain in premature infants can lead to adverse neurocognitive and neurodevelopmental outcomes [39]. Premature infants can experience extended bouts of hypoxia [40, 41], and MRI studies have demonstrated altered connectivity in ex-premature infants [42, 43]. Our findings suggest 


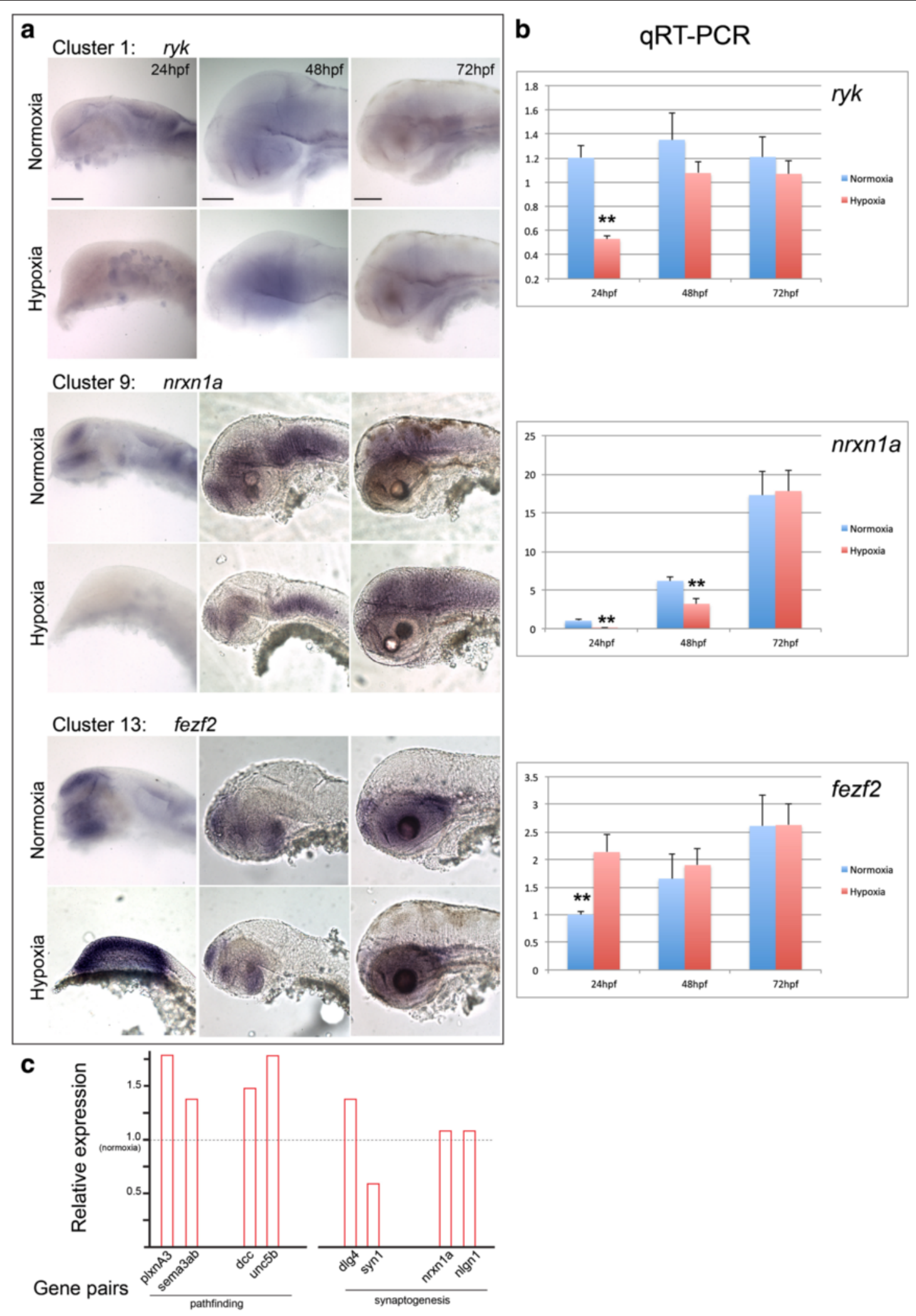

Fig. 6 (See legend on next page.) 


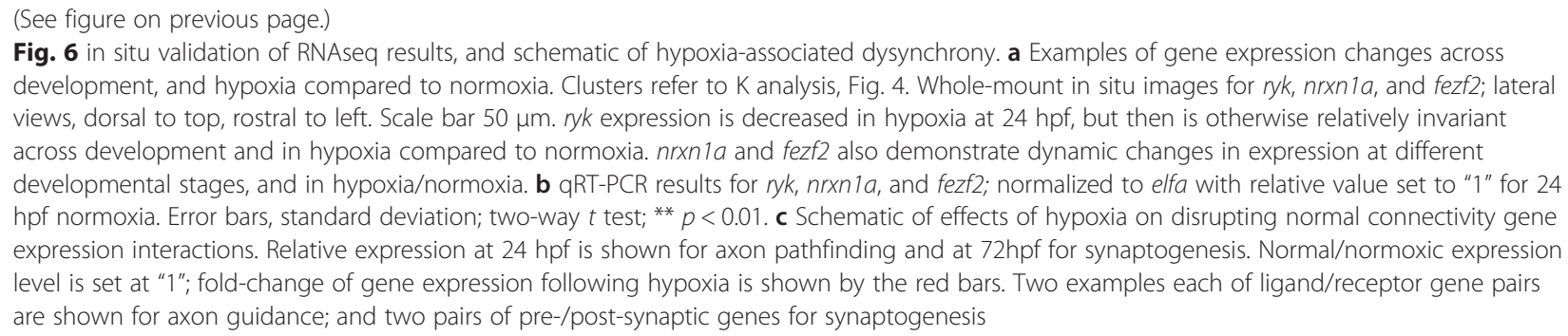

that certain key genetic pathways may be affected in premature infants by hypoxia.

In summary, our data suggests two central findings concerning the effects of hypoxia on CNS connectivity development. First, that the major effects of hypoxia are due to a dysynchrony of gene expression; and second, that hypoxia disproportionately affects a subset of connectivity genes. These results should lead to further investigations on why certain genes are prone to the effects of hypoxia, and what effects those gene responses have on the developing CNS.

\section{Methods}

\section{Ethics statement}

Zebrafish experiments were approved and performed under guidelines from the University of Utah Institutional Animal Care and Use Committee (IACUC), and regulated under federal law (the Animal Welfare Act and Public Health Services Regulation Act) by the U.S. Department of Agriculture (USDA) and the Office of Laboratory Animal Welfare at the $\mathrm{NIH}$, and accredited by the Association for Assessment and Accreditation of Laboratory Care International (AAALAC).

\section{Fish stocks and embryo raising}

Adult fish were bred according to standard methods [44]. Strain AB was used for all experiments. Embryos were raised at $28.5{ }^{\circ} \mathrm{C}$ in E3 embryo medium. For in situ staining embryos were fixed in $4 \%$ paraformaldehyde (PFA) in PBS overnight $(\mathrm{O} / \mathrm{N})$ at $4{ }^{\circ} \mathrm{C}$, washed briefly in PBS with $0.1 \%$ Tween-20, dehydrated, and stored in $100 \% \mathrm{MeOH}$ at $-20{ }^{\circ} \mathrm{C}$ until use.

\section{Hypoxia}

Embryonic zebrafish were placed in a sealed plexiglass chamber connected via a controller that monitored and adjusted nitrogen gas flow to a desired $\mathrm{pO}_{2}$ set point (Biospherix Ltd.) (full details in [12]). All solutions were pre-equilibrated to either normoxia or hypoxia for at least $4 \mathrm{~h}$ before use, and embryos were transferred into and out of pre-equilibrated solutions. Following hypoxia exposure, embryos were returned to media kept in normoxic conditions. Timed staging was used to determine age at fixation for analyses and RNA collection.
RNA extraction, CDNA preparation, and sequencing

Embryo heads were manually prepared by dissection with a tungsten needle. Following dissection, embryo heads were triturated in Trizol with a 25-gauge needle on a $1 \mathrm{~mL}$ syringe. RNA was prepared with a miRNeasy kit (Qiagen). Quality of total RNAs was checked using a NanoDrop 2000 Spectrometer and an Agilent 2100 Bioanalyzer. RNA integrity number (RIN) from the Bioanalyzer ranged from 7.2 to 9.7 (average 9.1; median 9.4). Library preparation and sequencing was done at the University of Utah Microarray and Genomic Analysis Shared Resource on a Hiseq 2000 machine and processed using the Cassava 1.8 pipeline. We generated 50 base single-end reads from the RNA libraries. 30 samples were multiplexed across 5 lanes of a single flow cell, for a total of 6 samples per lane. We generated on average 25,073,213 reads per biological replicate. Raw sequence data has been uploaded to the Sequence Read Archive under BioProject PRJNA293106 (http://www.ncbi.nlm.nih.gov/bioproject/PRJNA293106) where the per-sample sequencing yield is available.

Total RNA from zebrafish was collected at 24hpf, $36 \mathrm{hpf}, 48 \mathrm{hpf}, 60 \mathrm{hpf}$, and $72 \mathrm{hpf}$; with biological triplicates for each stage (time-point) and for each experimental condition. For RNAseq and qRT-PCR, pools of $\sim 30 \mathrm{em}-$ bryos for each sample (stage and experimental condition) were collected.

\section{RNAseq alignment}

Raw sequence data quality was checked using FastQC version 0.10.1 (http://www.bioinformatics.babraham.ac.uk/projects/fastqc/). No sequence quality issues were found so no adapter or quality trimming was performed prior to genome alignment. We did perform adapter trimming of the RNA reads during alignment to the zebrafish $\mathrm{Zv} 9$ genome and known splice sites using the novoalign aligner (www.novocraft.com). Mapping was done using Novoalign version 2.0.8 (Novocraft Inc, http://www.novocraft.com) with default parameters except output was set to SAM format, and one random alignment was chosen for fragments aligning to multiple locations ( $-\mathrm{r}$ Random). Reads were aligned to the $\mathrm{Zv} 9$ zebrafish build [20] with splice junctions derived from the UCSC Ensembl 


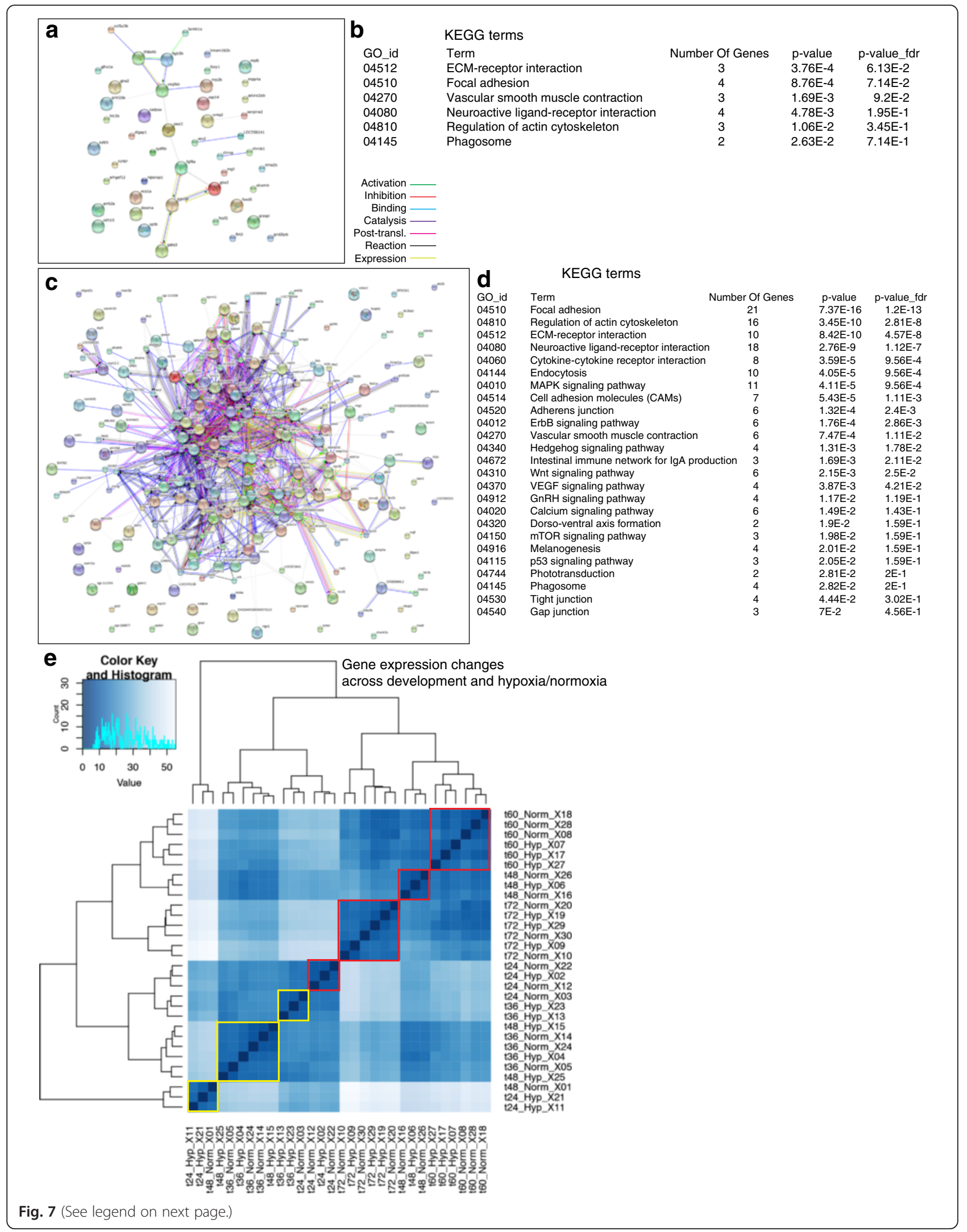


(See figure on previous page.)

Fig. 7 Protein-Protein Interactions Network. a STRING analysis of most significant (adjusted $p<0.05$ ) genes interactions, $n=57$; color key for interaction type is shown to the right (Additional file 10: Figure S2 shows enlarged picture and fonts). $\mathbf{b}$ KEGG analysis of the most common pathways for pathways with 2 or more genes. c STRING analysis with relaxed criteria (unadjusted $p<0.05$ ), $n=244$ (Additional file 10: Figure S2 shows enlarged picture and fonts). $\mathbf{d}$ KEGG analysis of pathways using relaxed criteria for pathways with 2 or more genes. e Heat-map profile of gene expression changes across development and experimental conditions. Red boxes show clusters in which all the groups had the same age but had both hypoxic and normoxic samples. Yellow boxes show clusters in which both the age and the experimental condition varied. At younger ages hypoxia is noted to have a larger effect by causing differential clustering based on the presence of hypoxia/normoxia (yellow boxes) rather than based solely on age (the red boxes)

refFlat gene table (http://genome.ucsc.edu/goldenpath/ gbdDescriptionsOld.html\#RefFlat).

\section{RNA preparation in normoxia and hypoxia during development}

For hypoxia, we placed developing embryos in preconditioned media in a sealed plexiglass chamber set to a reduced $\mathrm{O}_{2}$ concentration [12] for 12 h periods. Immediately following hypoxia (or normoxia) embryos were sacrificed and RNA collected. Based on our previous studies of the role of hypoxia on CNS development and effects on lethality [12], we used $1 \% \mathrm{pO}_{2}$ for ages up to 48 hpf (hours post-fertilization); and $4 \% \mathrm{pO}_{2}$ for older ages. At the time-point for collection embryos were dissected and only head tissue was used for subsequent RNA preparation.

\section{Transcript/genomic alignment}

Following sequencing, RNA reads were aligned to the zebrafish genome and splice junctions with genome build Zv9 [20]. A total of 31,860 different transcripts, obtained from the UCSC Ensembl RefFlat table, were tested for differential expression using DESeq2.

For the majority of our subsequent analyses, we restricted our analysis to 1270 genes with known or likely roles in the development of CNS connectivity. The 1270 genes were identified based on the gene ontology (GO) terms "axon guidance" or "synapse". The search for genes meeting the GO criteria was performed separately in the zebrafish and human genome assemblies; the lists of genes were merged and the zebrafish orthologs were then substituted for any genes only present on the list from the human genome due to incomplete annotation of the zebrafish genome. The file was then manually reviewed for omissions, and the following genes with known (published) roles in CNS connectivity development were manually added: apoeb, appb, ctbp2, dlg4, ephrinB2a, foxP2, fzd3a, lamb1a, lamc1, mnx1, nav1, neo1, sema3b, sparc, sparcl1, tgfbr2, thbs1, thbs2, and thbs4.

\section{$\mathrm{K}$ cluster and statistical analyses}

Gene expression analysis was performed using the Useq 7.8.1 software package with default parameters [45].
Differentially expressed genes were defined as genes with a false discovery rate less than 0.1 and more than a 2-fold expression change.

K-means clustering on $\log _{2}$-transformed FPKM values was performed using $R$ version 3.1. The value of $K$ was chosen by plotting the total within-cluster variance as a function of $\mathrm{K}$, identifying the minimum $\mathrm{K}$ for which the total within-cluster variance was less than $20 \%$ of the total variance and the variance curve showed a marked decrease in slope as $\mathrm{K}$ increased. For normoxic samples $\mathrm{K}=10$ met these criteria. For samples with normoxic and hypoxic data sets, $\mathrm{K}=14$ was selected.

\section{Pathway analysis}

KEGG and GO enrichment was analyzed using DAVID (http://david.abcc.ncifcrf.gov) [46, 47] functional annotation tools with default parameters. Each cluster of genes identified by K-means clustering was annotated at the DAVID site. The KEGG pathway/gene cluster associations were hierarchically clustered using the $-\log _{10}(\mathrm{p})$ value of the pathway enrichment. Clustering was performed in $\mathrm{R}$ version 3.1 using gplots (http://cran.r-project.org/web/packages/gplots/index.html).

Pathway Commons Analysis was performed using PCViz (http://www.pathwaycommons.org/pcviz/) [24].

\section{Principal Component Analysis}

The principal components analysis was performed in $\mathrm{R}$ (version 3.1.2) using the prcomp function (https://stat.ethz.ch/R-manual/R-patched/library/stats/html/ prcomp.html). Log-scale FPKM values were centered and scaled in the prcomp function prior to analysis.

\section{in situ hybridization}

Whole-mount in situ labeling for ryk, neurexin1a, and fezf2 was performed as previously described [48]. Briefly, embryos were fixed overnight in $4 \%$ paraformaldehyde/ PBS at $4{ }^{\circ} \mathrm{C}$ and stored in $100 \%$ methanol. Embryos were permeablilized with $10 \mu \mathrm{g} / \mathrm{ml}$ proteinase $\mathrm{K}$, and then digoxigenin-labeled probes were hybridized and detected with alkaline phosphatase-conjugated antidigoxigenin Fab fragment (1:5000, Roche) and BM purple alkaline phosphatase substrate. 30 or more embryos were analyzed for each probe and condition. Probes 
were generated by PCR amplification of genomic exon coding sequence and cloned into pCR4-TOPO (Invitrogen). Primers were (forward; reverse; listed $5^{\prime}$ to 3 '): $r y k$, CGCATACGGTACAGTGGAGA; TTGACCTCTTCCTC GCTCAT; neurexin1a, CTGCGAAAGCGAAATGAGTT; GACTCCTCCATTCAGGCAAA; and fezf2, GCAAGCCAAGGCTTTAATGA; TTCCCGTCTGAAGAGCAGTT.

\section{Microscopy and image analysis}

Following in situ staining, embryos were transferred step-wise into $80 \%$ glycerol $/ 20 \%$ PBST, mounted on a glass slide with a $\# 0$ coverslip placed over a well made using electrical tape, and imaged on a compound light microscope. Illustrations were composed with Adobe Photoshop and Illustrator.

\section{Quantitative RT-PCR}

New samples were prepared separately for the RT-PCR with three independent biological replicates. Real-time PCR was performed on an ABI PRISM 7900HT (Applied Biosystems) with SYBR Green fluorescence label. qPCR for ryk, nrnxla, fezf2, atoh1a, arxa, col5A3a, htr3a and $z i c 2 b$ was performed on cDNA prepared from $24 \mathrm{hpf}$, $48 \mathrm{hpf}$ and $72 \mathrm{hpf}$ embryos. Each reaction was performed in triplicate and the mean of replicates was calculated; results were normalized to the mRNA level of each gene in normoxic embryos at $24 \mathrm{hpf}$ with elongation fator I alpha (elfa) transcript levels as a control. Primers were as follows (shown as 5' to 3', forward; reverse): ryk, ACACCTGTCACAAGTTATCCAT, AGACGTCTCGAA GTGTGACT; nrxn1a, CCACAACTCCACAGGACGAT, GGGAAGTGGTGGGTGAGCT; fezf2, GGCAACTGGAC CAAATCGTG, AGTGCGCGTTGAAGACCTTT; atoh1a, GAGAGTTCTCGCCTCACTCG, TCCGGCGGTGTGT TTTCTTA; arxa, GACAACCGAAGTCACCTCCAA, TC TAGGTGCTCGTGAAACCC; col5A3a, TCAGGACAGCAGTCCCTCTAC, TTAATCGGCCTCTCCTGCTTTG; htr3a, TGGAGTTCAGCCTGCATCAC, ACTGAGCAA TCCACCACTGT; $z i c 2 b$, AACACATGAAGGTTCACGA GGA, AGAATCAGGCGACAAGGTGC; elfa, CTTCTCA GGCTGACTGTGC, CCGCTAGCATTACCCTCC. Reactions were run with the following conditions: $95{ }^{\circ} \mathrm{C}$ for 10 min and 40 cycles of $95{ }^{\circ} \mathrm{C} 20 \mathrm{~s} / 60{ }^{\circ} \mathrm{C} 20 \mathrm{~s} / 72{ }^{\circ} \mathrm{C} 40 \mathrm{~s}$. The relative levels of each target mRNA were normalized to the mRNA level of each gene in normoxic embryos at $24 \mathrm{hpf}$ with elongation factor 1- $\alpha$ (eef1a1) transcript levels as a control using the $2-\Delta \Delta \mathrm{CT}$ method. For statistical analysis, student's $t$ test was used to compare normoxia and hypoxia groups.

\section{Availability of supporting data}

The data sets supporting the results of this article are included within the article and its additional files. The RNAseq data is available at SRP062493.

\section{Additional files}

Additional file 1: List of final 1270 genes for analysis (Ensembl gene ID listed). These genes were chosen based on known or likely roles in CNS connectivity development. (XLS $99 \mathrm{~kb}$ )

Additional file 2: List of K-means cluster analysis of connectivity genes across development during normoxia. Relative log2 fold change compared to the developmental average is provided. (XLSX $171 \mathrm{~kb}$ )

Additional file 3: List of K-means cluster analysis of connectivity genes across development during hypoxia. Relative log2 fold change compared to the developmental average is provided. (XLSX $197 \mathrm{~kb}$ )

Additional file 4: Figure S1. GO analysis from Fig. $3 \mathrm{D}$ and $\mathrm{H}$, with $\mathrm{GO}$ terms displayed. Developmental profiles of connectivity genes $(n=1270)$, organized as K-means clusters with groups of genes showing similar expression pattern profiles across development. A) GO pathway analysis of K clusters in normoxia. B) GO pathway analysis of K clusters comparing hypoxia to normoxia. (JPG $1354 \mathrm{~kb}$ )

Additional file 5: List of $\mathrm{K}$ means cluster analysis of connectivity genes across development during hypoxia, after averaging relative expression across development in both hypoxia and normoxia. Relative log2 fold change compared to the average is provided. (XLSX $257 \mathrm{~kb}$ )

Additional file 6: Data sheets of qRT-PCR results for atoh1a, arx, col5A3, htr3a, and zic2b. First tab for each gene has raw data; second tab has analysis; third tab has statistical analyses. (XLSX 166 kb)

Additional file 7: List of all genes analysis (Ensembl gene ID listed). Cluster assignment is shown; and relative counts in normoxia and hypoxia at different times. (XLSX $261 \mathrm{~kb}$ )

Additional file 8: Data sheets of qRT-PCR results for $r y k, n r \times n 1 a$, and fezf2. First tab for each gene has raw data; second tab has analysis; third tab has statistical analyses. (XLSX $109 \mathrm{~kb}$ )

Additional file 9: Data sheets of connectivity genes; analyzed for unadjusted, adjusted for multiple comparisons, and FDR, across development and experimental conditions (comparing normoxia to hypoxia). (XLSX 3494 kb)

Additional file 10: Figure S2. Larger file and font sizes showing gene names for the Protein-Protein Interactions Network. A) STRING analysis of most significant (adjusted $p<0.05$ ) genes interactions, $n=57$; color key for interaction type is shown to the right. B) STRING analysis with relaxed criteria (unadjusted $p<0.05$ ), $n=244$. (JPG $2997 \mathrm{~kb}$ )

\section{Abbreviations}

ADHD: attention-deficit hyperactivity disorder; CNS: central nervous system; FACS: fluorescence-activated cell sorting; FDR: false discovery rate;

FPKM: fragments per kilobase of transcript per million fragments mapped; GO: gene ontology; hif: hypoxia-inducible factor; hpf: hours post-fertilization; KEGG: Kyoto encyclopedia of genes and genomes; MRI: magnetic resonance imaging; PCA: principal component analysis; RT-PCR: reverse transcription polymerase chain reaction; TRAP: translating ribosomal affinity purification.

\section{Competing interests}

The authors declare that they have no competing interests.

\section{Authors' contributions}

BM performed the data alignment and analysis. TJS and JLB collected samples and performed the hypoxia experiments. JG, TD and JHS performed experimental validation of results. BM and JLB designed the study. JLB conceived of the study and participated in its design and coordination. All authors helped draft the manuscript, and read and approved the final manuscript.

\section{Acknowledgements}

This work was supported by NIH grant DP2 MH100008, R21 MH107039, and a March of Dimes Research Grant to JLB. BM was supported in part by $\mathrm{NIH}$ / $\mathrm{NCl}$ grant P30 CA042014. 


\section{Author details}

'Bioinformatics Shared Resource, Huntsman Cancer Institute, Salt Lake City, USA. ${ }^{2}$ Department of Pediatrics, University of Utah, 295 Chipeta Way, 84108 Salt Lake City, UT, USA. ${ }^{3}$ Department of Neurobiology and Anatomy, University of Utah School of Medicine, Salt Lake City, UT, USA.

Received: 5 January 2016 Accepted: 22 April 2016

Published online: 04 May 2016

\section{References}

1. Bass UL, Corwin M, Gozal D, Moore C, Nishida H, Parker S, et al. The effect of chronic or intermittent hypoxia on cognition in childhood: a review of the evidence. Pediatrics. 2004;114(3):805-16.

2. Gozzo Y, Vohr B, Lacadie C, Hampson M, Katz KH, Maller-Kesselman J, et al. Alterations in neural connectivity in preterm children at school age. Neuroimage. 2009;48(2):458-63. Pubmed Central PMCID: 2775072

3. Mullen KM, Vohr BR, Katz KH, Schneider KC, Lacadie C, Hampson M, et al. Preterm birth results in alterations in neural connectivity at age 16 years. Neuroimage. 2011;54(4):2563-70. Pubmed Central PMCID: 3020252.

4. Haynes RL, Borenstein NS, Desilva TM, Folkerth RD, Liu LG, Volpe JJ, et at. Axonal development in the cerebral white matter of the human fetus and infant. J Comp Neurol. 2005;484(2):156-67.

5. ten Donkelaar HJ. Major events in the development of the forebrain. Eur Morphol. 2000:38(5):301-8.

6. Kostovic I, Jovanov-Milosevic N. The development of cerebral connections during the first 20-45 weeks' gestation. Semin Fetal Neonatal Med. 2006; 11(6):415-22.

7. Geschwind DH, Levitt P. Autism spectrum disorders: developmental disconnection syndromes. Curr Opin Neurobiol. 2007;17(1):103-11.

8. Baribeau DA, Anagnostou E. A comparison of neuroimaging findings in childhood onset schizophrenia and autism spectrum disorder: a review of the literature. Frontiers in psychiatry. 2013;4:175. Pubmed Central PMCID: 3869044.

9. Shepherd GM. Corticostriatal connectivity and its role in disease. Nat Rev Neurosci. 2013;14(4):278-91. Pubmed Central PMCID: 4096337.

10. Akil H, Martone ME, Van Essen DC. Challenges and opportunities in mining neuroscience data. Science. 2011;331(6018):708-12. Pubmed Central PMCID: 3102049.

11. Polleux F. Genetic mechanisms specifying cortical connectivity: let's make some projections together. Neuron. 2005;46(3):395-400.

12. Stevenson TJ, Trinh T, Kogelschatz C, Fujimoto E, Lush ME, Piotrowski T, et al. Hypoxia disruption of vertebrate CNS pathfinding through ephrinB2 Is rescued by magnesium. PLoS Genet. 2012;8(4):e1002638. Pubmed Central PMCID: 3325188

13. Pocock R, Hobert O. Oxygen levels affect axon guidance and neuronal migration in Caenorhabditis elegans. Nat Neurosci. 2008;11(8):894-900.

14. Curristin SM, Cao A, Stewart WB, Zhang H, Madri JA, Morrow JS, et al. Disrupted synaptic development in the hypoxic newborn brain. Proc Natl Acad Sci U S A. 2002;99(24):15729-34. Pubmed Central PMCID: 137784

15. Sharp FR, Bernaudin M. HIF1 and oxygen sensing in the brain. Nat Rev Neurosci. 2004;5(6):437-48.

16. Tomita S, Ueno M, Sakamoto M, Kitahama Y, Ueki M, Maekawa N, et al. Defective brain development in mice lacking the Hif-1alpha gene in neural cells. Mol Cell Biol. 2003:23(19):6739-49. Pubmed Central PMCID: 193947.

17. Phatak NR, Stankowska DL, Krishnamoorthy RR. Transcription Factor Brn-3b Overexpression Enhances Neurite Outgrowth in PC12 Cells Under Condition of Hypoxia. Cell Mol Neurobiol. 2015.

18. Lange J, Yafai $Y$, Noack A, Yang XM, Munk AB, Krohn S, et al. The axon guidance molecule Netrin-4 is expressed by Muller cells and contributes to angiogenesis in the retina. Glia. 2012;60(10):1567-78.

19. O'Driscoll CM, Gorman AM. Hypoxia induces neurite outgrowth in PC12 cells that is mediated through adenosine A2A receptors. Neuroscience. 2005:131(2):321-9.

20. Howe K, Clark MD, Torroja CF, Torrance J, Berthelot C, Muffato M, et al. The zebrafish reference genome sequence and its relationship to the human genome. Nature. 2013;496(7446):498-503. Pubmed Central PMCID: 3703927

21. Phillips JB, Westerfield M. Zebrafish models in translational research: tipping the scales toward advancements in human health. Dis Model Mech. 2014; 7(7):739-43. Pubmed Central PMCID: 4073263.
22. Stewart AM, Braubach O, Spitsbergen J, Gerlai R, Kalueff AV. Zebrafish models for translational neuroscience research: from tank to bedside. Trends Neurosci. 2014;37(5):264-78. Pubmed Central PMCID: 4039217.

23. Love MI, Huber W, Anders S. Moderated estimation of fold change and dispersion for RNA-seq data with DESeq2. Genome Biol. 2014;15(12):550. Pubmed Central PMCID: 4302049.

24. Cerami EG, Gross BE, Demir E, Rodchenkov I, Babur O, Anwar N, et al. Pathway Commons, a web resource for biological pathway data. Nucleic Acids Res. 2011;39(Database issue):D685-90. Pubmed Central PMCID: 3013659.

25. Yaron A, Huang PH, Cheng HJ, Tessier-Lavigne M. Differential requirement for Plexin-A3 and -A4 in mediating responses of sensory and sympathetic neurons to distinct class 3 Semaphorins. Neuron. 2005:45(4):513-23.

26. Moore SW, Tessier-Lavigne M, Kennedy TE. Netrins and their receptors. Adv Exp Med Biol. 2007;621:17-31.

27. Yamagata M, Sanes JR, Weiner JA. Synaptic adhesion molecules. Curr Opin Cell Biol. 2003;15(5):621-32.

28. Szklarczyk D, Franceschini A, Wyder S, Forslund K, Heller D, Huerta-Cepas J, et al. STRING v10: protein-protein interaction networks, integrated over the tree of life. Nucleic Acids Res. 2015;43(Database issue):D447-52. Pubmed Central PMCID: 4383874

29. Drobyshevsky A, Jiang R, Lin L, Derrick M, Luo K, Back SA, et al. Unmyelinated axon loss with postnatal hypertonia after fetal hypoxia. Ann Neurol. 2014;75(4):533-41.

30. Palaisa KA, Granato M. Analysis of zebrafish sidetracked mutants reveals a novel role for Plexin A3 in intraspinal motor axon guidance. Development 2007;134(18):3251-7.

31. Bustos FJ, Varela-Nallar L, Campos M, Henriquez B, Phillips M, Opazo C, et al. PSD95 suppresses dendritic arbor development in mature hippocampal neurons by occluding the clustering of NR2B-NMDA receptors. PLoS One. 2014;9(4):e94037. Pubmed Central PMCID: 3976375.

32. Baldelli P, Fassio A, Valtorta F, Benfenati F. Lack of synapsin I reduces the readily releasable pool of synaptic vesicles at central inhibitory synapses. $J$ Neurosci. 2007:27(49):13520-31

33. Santiago C, Bashaw GJ. Transcription factors and effectors that regulate neuronal morphology. Development. 2014;141(24):4667-80. Pubmed Central PMCID: 4299270.

34. Doyle JP, Dougherty JD, Heiman M, Schmidt EF, Stevens TR, Ma G, et al. Application of a translational profiling approach for the comparative analysis of CNS cell types. Cell. 2008;135(4):749-62. Pubmed Central PMCID: 2763427.

35. Dengler VL, Galbraith MD, Espinosa JM. Transcriptional regulation by hypoxia inducible factors. Crit Rev Biochem Mol Biol. 2014;49(1):1-15. Pubmed Central PMCID: 4342852.

36. Trollmann R, Gassmann M. The role of hypoxia-inducible transcription factors in the hypoxic neonatal brain. Brain Dev. 2009;31(7):503-9.

37. Rytkonen KT, Prokkola JM, Salonen V, Nikinmaa M. Transcriptional divergence of the duplicated hypoxia-inducible factor alpha genes in zebrafish. Gene. 2014:541(1):60-6.

38. Yee Koh M, Spivak-Kroizman TR, Powis G. HIF-1 regulation: not so easy come, easy go. Trends Biochem Sci. 2008;33(11):526-34.

39. Horwood L, Mogridge N, Darlow BA. Cognitive, educational, and behavioural outcomes at 7 to 8 years in a national very low birthweight cohort. Arch Dis Child Fetal Neonatal Ed. 1998;79(1):F12-20. Pubmed Central PMCID: 1720817.

40. Noori S, Stavroudis TA, Seri I. Systemic and cerebral hemodynamics during the transitional period after premature birth. Clin Perinatol. 2009:36(4): 723-36. v.

41. Martin RJ, Wang K, Koroglu O, Di Fiore J, Kc P. Intermittent hypoxic episodes in preterm infants: do they matter? Neonatology. 2011;100(3):303-10. Pubmed Central PMCID: 3252018.

42. Estep ME, Smyser CD, Anderson PJ, Ortinau CM, Wallendorf M, Katzman CS, et al. Diffusion tractography and neuromotor outcome in very preterm children with white matter abnormalities. Pediatr Res. 2014;76(1):86-92. Pubmed Central PMCID: 4062577

43. Pandit AS, Robinson E, Aljabar P, Ball G, Gousias IS, Wang Z, et al. Wholebrain mapping of structural connectivity in infants reveals altered connection strength associated with growth and preterm birth. Cereb Cortex. 2014;24(9):2324-33.

44. Kimmel CB, Ballard WW, Kimmel SR, Ullmann B, Schilling TF. Stages of embryonic development of the zebrafish. Dev Dyn. 1995;203(3):253-310. 
45. Nix DA, Courdy SJ, Boucher KM. Empirical methods for controlling false positives and estimating confidence in ChIP-Seq peaks. BMC bioinformatics. 2008:9:523. Pubmed Central PMCID: 2628906.

46. da Huang W, Sherman BT, Lempicki RA. Systematic and integrative analysis of large gene lists using DAVID bioinformatics resources. Nat Protoc. 2009; 4(1):44-57.

47. Huang DW, Sherman BT, Zheng X, Yang J, Imamichi T, Stephens R, Lempicki RA. Extracting Biological Meaning from Large Gene Lists with DAVID. Curr Protoc Bioinform. 2009;27:13.11:13.11.1-13.11.13.

48. Bonkowsky JL, Chien CB. Molecular cloning and developmental expression of foxP2 in zebrafish. Dev Dyn. 2005:234(3):740-6.

Submit your next manuscript to BioMed Central and we will help you at every step:

- We accept pre-submission inquiries

- Our selector tool helps you to find the most relevant journal

- We provide round the clock customer support

- Convenient online submission

- Thorough peer review

- Inclusion in PubMed and all major indexing services

- Maximum visibility for your research

Submit your manuscript at www.biomedcentral.com/submit
Biomed Central 